
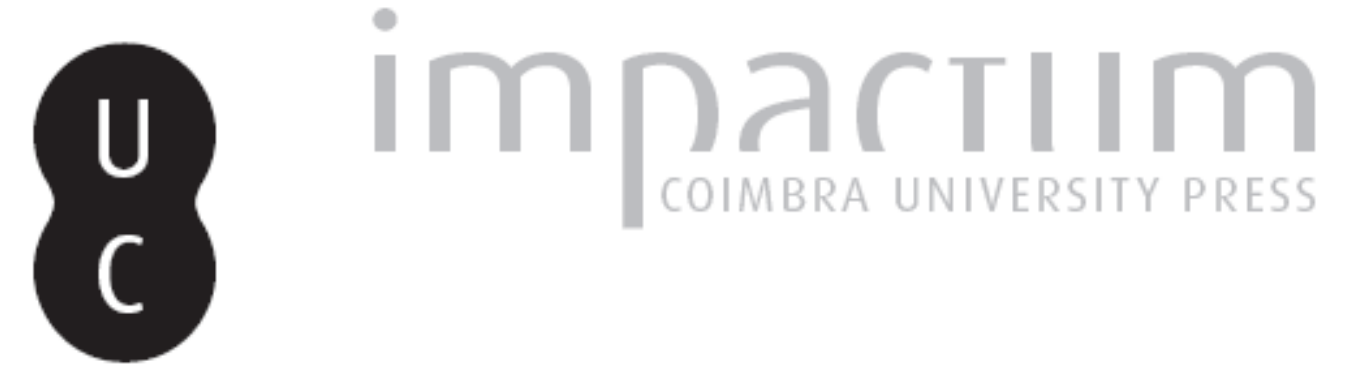

\title{
Los macheteros y el rol de la prensa en la lucha de liberación nacional en Puerto Rico
}

Autor(es): $\quad$ Pesante, Francisco

Publicado por: Imprensa da Universidade de Coimbra

URL persistente:

URI:http://hdl.handle.net/10316.2/39633

DOI:

DOI:http://dx.doi.org/10.14195/2183-5462_29_7

Accessed : $\quad$ 26-Apr-2023 15:03:39

A navegação consulta e descarregamento dos títulos inseridos nas Bibliotecas Digitais UC Digitalis, UC Pombalina e UC Impactum, pressupõem a aceitação plena e sem reservas dos Termos e Condições de Uso destas Bibliotecas Digitais, disponíveis em https://digitalis.uc.pt/pt-pt/termos.

Conforme exposto nos referidos Termos e Condições de Uso, o descarregamento de títulos de acesso restrito requer uma licença válida de autorização devendo o utilizador aceder ao(s) documento(s) a partir de um endereço de IP da instituição detentora da supramencionada licença.

Ao utilizador é apenas permitido o descarregamento para uso pessoal, pelo que o emprego do(s) título(s) descarregado(s) para outro fim, designadamente comercial, carece de autorização do respetivo autor ou editor da obra.

Na medida em que todas as obras da UC Digitalis se encontram protegidas pelo Código do Direito de Autor e Direitos Conexos e demais legislação aplicável, toda a cópia, parcial ou total, deste documento, nos casos em que é legalmente admitida, deverá conter ou fazer-se acompanhar por este aviso.

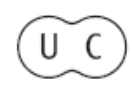




\section{Media Jornalismo}

\section{MÉDIA E COLONIALISMO(S)}




\section{LOS MACHETEROS Y EL ROL DE LA PRENSA EN LA LUCHA DE LIBERACIÓN NACIONAL EN PUERTO RICO}

\section{THE MACHETEROS AND THE ROLE OF THE PRESS IN THE NATIONAL LIBERATION STRUGGLE IN PUERTO RICO}

\section{FRANCISCO J. PESANTE GONZÁLEZ}

UNIVERSITY OF PHOENIX-PUERTO RICO CAMPUS, ESTUDIANTE DOCTORAL: CENTRO

DE ESTUDOS DE PUERTO RICOY EL CARAIBE SAN JUAN, PUERTO RICO

GUAYNAB0, PR00968, PUERT0 RIC0

FRANCISCOPESANTE@YAHOO.COM

Resumen:

El siguiente artículo recapitula parte la cobertura de prensa de la detención, el encarcelamiento y el procesamiento de los miembros de la organización separatista clandestina puertorriqueña Los Macheteros. Con la intensión de acentuar la importancia de la prensa independiente y los organismos de solidaridad política para romper el cerco informático por parte de las autoridades o sectores de la prensa comercial (mass media) que reproducen los discursos hegemónicos del estado. Además de reconocer su valor como fuente histórica. También se reseña algunos acontecimientos en los que las autoridades coloniales interfirieron con el derecho a la libertad de prensa, al interferir con algunos periodistas que trabajaron de cerca las historias relacionadas a Los Macheteros. Eventos que permiten reflexionar sobre la reproducción o desafíos de las posturas oficiales articuladas por las autoridades contra los miembros de la organización clandestina y la cobertura de los acontecimientos por la prensa.

\section{Palabras claves}

Puerto Rico, colonialismo, independencia, robo a la Wells Fargo, Daisy Sánchez, libertad de prensa, Filiberto Ojeda Ríos, Movimientos de liberación nacional

ABstract:

The following paper reviews the press coverage of the arrest, detention and prosecution of members of the clandestine Puerto Rican separatist group Los Macheteros. We emphasize the relevance of the independent press and political solidarity groups' defiance of the official stance against the anticolonial armed movement and the mass media reproduction of the hegemonic discourse. Pointing the authorities' interference with the right to freedom of the press. As well as pointing out the writter press value as a rich historical research reference.

KEYWORDS

Puerto Rico, Colonialism, Independence, Wells Fargo Robbery, Daisy Sánchez, Freedom of the Press, Filiberto Ojeda Ríos, National liberation movements 
InTRODUCCIÓN

A lo largo del siglo veinte, la isla de Puerto Rico tuvo varios episodios de luchas políticas por lograr la independencia política de Estados Unidos. Estados Unidos adquirió la isla de Puerto Rico como colonia bajo el Tratado de París de 1898, el cual formalizó su pacto con España tras la conclusión de la Guerra Hispanoamericana. Uno de los últimos episodios de las luchas separatistas de Puerto Rico contra los Estados Unidos se llevó a cabo en el contexto de la proliferación de los movimientos de liberación nacional en África y el mediano Oriente. En el caso de Puerto Rico, esta secuela de luchas alcanzó su apogeo entre las décadas de 1970 y 1980, tanto en la isla como en los Estados Unidos continentales, por miembros de la diáspora puertorriqueña.

En el caso bajo estudio se utilizó, entre otras fuentes, la prensa escrita para la reconstrucción de eventos en torno a la operación de una organización de liberación nacional Los Macheteros, se analizó el rol de los medios de prensa independientes (fuera de los circuitos de la prensa comercial), así como las campañas de excarcelación en los procesos legales y la lucha por legitimidad entre el Estado y los miembros de la organización clandestina separatista. En este ejercicio también se apuntó el rol de algunos reporteros que, sin tomar bando en el asunto político, en su rol periodístico fueron sujetos a los aparatos represivos del estado colonial. Afectando el derecho a la libertad de prensa cobijada tanto en la Constitución coIonial como en la Carta Magna de la metrópoli estadounidense.

\section{LOS RETOS DE ESTUDIAR LAS ORGANIZACIONES DE LIBERACIÓN NACIONAL}

Por su naturaleza clandestina, el estudio de las organizaciones de liberación nacional es un reto que requiere mucha creatividad.

En mi experiencia, eventos como la detención, el encarcelamiento y procesamiento judicial de los miembros de estas organizaciones, captados en los documentos legales derivados del proceso, ha sido una forma de poder lograr una aproximación a conocer quiénes fueron los miembros de estas organizaciones, cuál fue su ideología, su base ideológica y características operacionales de la organización, así como los métodos usados por el Estado para manejar las investigaciones y procesamiento judicial de imputados a pertenecer a estas organizaciones.

Reconociendo los sesgos ideológicos que pueden encontrarse en las fuentes mencionadas en el párrafo anterior, otra fuente que conviene consultar para el conocimiento de las organizaciones de liberación nacional lo es la prensa. Esta recoge la crónica de los acontecimientos en torno a estas organizaciones que han reconocido el valor de estas plataformas mediáticas. Que en las modalidades de lucha adoptadas después de la Segunda Guerra Mundial han utilizado los comunicados de prensa para explicar sus motivos y la intensión de sus operativos. La prensa también ha cumplido una función de importancia en la reseña, no solamente de los operativos políticos conducidos por estas organizaciones. También en la reseña de acontecimientos como detención, el encarcelamiento y procesamiento de los miembros de estas organizaciones clandestinas. Bajo su muy bien conocidas líneas editoriales que hacen de la pretensión de objetividad en los eventos reseñados por la prensa, nada más que eso. Una pretensión. 
Luego de 5 años de procesos judiciales, antecedido por 3 años de litigios sobre libertades condicionadas y detenciones preventivas, en 1992 el proceso judicial contra Los Macheteros parecía encontrar un fin al proceso iniciado en 1985.

Los Macheteros como organización surgió a mediados de 1976. Esta organización política clandestina luchaba por la independencia de Puerto Rico del poder imperial de los Estadous Unidos y la construcción de un sistema político socialista. Entre 1976 y 1985 (en este último año cuando 15 de sus miembros fueron arrestados por las autoridades estadounidense), la organización se había adjudicado varios operativos. Entre éstos se puede destacar el ataque armado de diciembre de 1979 a un autobús militar, matando dos militares estadounidenses. En enero de 1981, llevaron a cabo la destrucción de nueve aviones de la Guardia Nacional de Estados Unidos establecidos en la base aérea del municipio de Carolina, Puerto Rico. Este último, junto al robo de $\$ 7$ millones a la compañía Wells Fargo en Connecticut, Estados Unidos, fueron sus operativos más grandes (Fernández, 1993: 87-88, 345-347).

Luego de los arrestos por las autoridades federales estadounidenses de los miembros de Los Macheteros en agosto de 1985, Ios operativos político-militares en Puerto Rico y Estados Unidos por parte de organizaciones independentistas que patrocinaban la lucha armada para la liberación nacional, prácticamente desaparecieron.

Los arrestos realizados por las autoridades del gobierno federal de los Estados Unidos en 1985 contra los miembros de Los Macheteros se extendió a Puerto Rico, Texas y México. Todos involucrados al robo de $\$ 7$ millones en Hartford, Connecticut ${ }^{1}$.

El robo habido en los depósitos de la compañía de acarreo de valores Wells Fargo en 1983, se había adjudicó originalmente al guardia de seguridad de la compañía Víctor Gerena. Sin embargo, un año después la organización radical independentista puertorriqueña Los Macheteros reclamó la autoría del delito, brindando pruebas al respecto (Fernández, 1993: 36-42) .

Esta revelación dio un giro a los acontecimientos. Lo que se había interpretado como un delito motivado por el lucro personal, fue redefinido por Los Macheteros como un acto político que se sumaba a una serie de ataques generados por la organización contra símbolos de los cuerpos de seguridad del Estado (tanto insular como estadounidense) en su lucha por adelantar la independencia política de Puerto Rico. ${ }^{3}$

Entre el comunicado de Los Macheteros adjudicándose la autoría del robo y el operativo de 1985, transcurrió casi un año. Ese fue el tiempo suficiente para que las autoridades federales se aseguraran de dar una respuesta fulminante a Los Macheteros.

1 Allanamientos y arrestos. Claridad (1985, septiembre 6). p. 2.; Delgado, A. La invasión del viernes 30. Claridad (1985, septiembre 6) p. 3.

2 Madden, R. L. Fiancée of \$7 Million Robbery Suspect is Arrested. The New York Times (1983, September 15). p. B 2; Madden, R.L. Wells Fargo Guard Acused of Stealing \$7 Million in Cash. The New York Times (1983, September 14) p. 1; Carrington, K.,Guard Robs \$8 Million of Wells Fargo. The Hartford Courant (1983, September 13). p. 1-A.

3 US v. Gerena, No. H-85-50 (D. Conn. Sep. 18, 1985), p. 37-38. Retirado de: http://www.latinamericanstudies.org/epb-macheteros.htm 
Los juicios habían sido precedidos por una larga lucha para la libertad bajo fianza, comenzada en septiembre de 1985, y extendiéndose hasta diciembre de 1986. Obteniendo una primera victoria en el caso de 13 de los 15 independentistas quienes lograron su libertad condicional. La excepción fueron Juan Segarra y Filiberto 0jeda quienes enfrentaron 31 y 34 meses respectivamente de detención preventiva.

Los litigios para la supresión de evidencia en general tuvieron un éxito limitado, teniendo como resultado la división del juicio en dos grupos, por iniciativa de la fiscalía. Esta iniciativa le permitió a la fiscalía separar a nueve de los acusados que no se veían afectados por la supresión de evidencia, para ser juzgados en un proceso posterior. Proceso que se extendió desde 1986 hasta fines de 1989. ${ }^{4}$

Eventualmente algunos acusados decidieron notificar mediante la prensa a los grupos de apoyo, la adopción de estrategias de defensa legal individual. Esto respondía a consideraciones muy prácticas. Cada acusado podía decidir: si aceptaba algún tipo de conocimiento, de simpatía o afiliación con Los Macheteros; plantear como teoría de la defensa haber participado de las actividades Probelitistas públicas de la organización, sin saber nada más allá; plantear que las acusaciones era una respuesta represiva del gobierno, exclusivamente por su ideología y afiliación independentista, entre otros argumentos. ${ }^{5}$

El Comité de Amigos y Familiares manifestó su parecer sobre la defensa individual de los acusados. Reconocía que las condiciones objetivas comunes de los acusados en la primera etapa (desde los arrestos hasta los procesos anteriores al juicio) facilitaban la adopción de posiciones unitarias. En este contexto las diferencias políticas quedaron en un segundo lugar, ante la necesidad de adquirir lo más pronto posible la libertad bajo fianza y denunciar la detención preventiva; las mociones para la supresión de evidencia; la impugnación de la composición del jurado y la promoción del traslado del caso judicial.

Según se fueron resolviendo los procesos iniciales antes de comienzo formal del juicio, se fueron acentuando las diferencias políticas entre los acusados. Los enfoques de los acusados sobre cómo encaminar la lucha por la independencia determinaron cómo se conduciría su defensa legal dentro de cada caso. Todo lo anterior incidió en la orientación de las campañas de apoyo existentes, así como en la participación y el consentimiento de los arrestados para ser representados por los distintos grupos de apoyo. A pesar de lo anterior, en el caso particular del Comité de Amigos y Familiares, así como posteriormente con Ofensiva '92, éstos demostraron su disponibilidad y completo respaldo a todos los presos políticos, siempre y cuando éstos no perjudicaran a sus colegas y aprovecharan el foro legal federal para denunciar la condición colonial de Puerto Rico ${ }^{6}$

Transcurridos casi siete años desde los arrestos de agosto de 1985 el resultado de los juicios contra los imputados por el robo de $\$ 7$ millones a la compañía de acarreo de valores Wells Fargo de Hartford, Connecticut fue el siguiente:

4 Anglada, R. 1988: ardua lucha legal en Hartford. Claridad (1988, diciembre 30). p. 4.

5 Anglada, R.. Hartford, segunda parte. Claridad (1989, enero 6) p.4.

6 Comité de Amigos y Familiares mantendrá respaldo, Parte 1. Claridad (1989, enero 6) p. 30;

Comité de Amigos y Familiares mantendrá respaldo, Parte 2, final.Claridad(1989, enero 12). p. 30. 
- Víctor Gerena, Norberto González Claudio y Avelino González Claudio - paradero desconocido (permanecían prófugos de la justicia).

- Juan Segarra Palmer - sentenciado a 55 años de prisión.

- Antonio Camacho Negrón - sentenciado a 15 años de prisión.

- Norman Ramírez Talavera, Roberto José Maldonado, Luz Berríos- sentenciado a 5 años de prisión.

- Carlos Ayes - absuelto.

- Ángel Díaz Ruiz y Elías Castro - le fueron retirados todos los cargos.

- Isaac Camacho - Sentenciado a tres años y ocho meses de prisión.

- Hilton Fernández Diamante y Orlando González Claudio - Enfrentaron sentencia de 5 años de prisión, una multa de 10 mil dólares y cinco años de probatoria.

- Jorge Farinacci -Sentenciado a tres años de prisión, una multa de 10 mil dólares y cinco años de probatoria.

- Ivonne Meléndez Carrión -tiempo servido (durante proceso de detención antes del proceso judicial) con servicio comunitario.

- Luis Colón Osorio - luego de 17 meses de arresto preventivo, dejó de comparecer al tribunal para retomar el clandestinaje. Arrestado en 1992, fue sentenciado a 1 año adicional de detención.

- Filiberto Ojeda Ríos - fue juzgado en ausencia, luego de violar las condiciones para su libertad bajo fianza. Sentenciado a 55 años. ${ }^{7}$

La prensa identificó como otra de las estrategias del gobierno de los Estados Unidos contra los independentistas su traslado a prisiones distantes de los familiares, a la vez de imponer una distancia considerable entre los mismos sentenciados, intensificando su aislamiento en las cárceles. ${ }^{8}$

Juan Segarra logró una reducción sustancial de su condena, luego del indulto del presidente William J. Clinton en 1999, por las presiones ejercidas por los grupos de solidaridad con los presos políticos. Siendo finalmente liberado en enero de 2004. ${ }^{9}$

En septiembre de 2005 Filiberto Ojeda Ríos, fue ejecutado por el Federal Bureau of Investigation (FBI), en su residencia de Puerto Rico. Al momento de su asesinato, se encontraba prófugo de la justicia (U.S. Attorney Office, 2010).

Por su parte, Ios hermanos Avelino y Norberto González Claudio fueron arrestados por el FBI en 2008 y 2011 respectivamente. Enfrentaron sentencias de 7 y 5 años. Esto por su vínculo con el robo de la Wells Fargo. (U.S. Attorney Office, 2012)

Al día de hoy, el paradero de Víctor Gerena sigue siendo desconocido

7 Pérez, M.. Han pasado 7 años... y la lucha sigue. Claridad (1992, abril 24) p. 5; Libertad. (1992, agosto 29). Tras siete años, caso "Wells Fargo" concluye con negociaciones y 2do juicio. Publicación del Comité Nacional Pro-Libertad Prisioneros Políticos y de Guerra Puertorriqueños. P.4.

8 Pérez, M. Sentenciado acusado en caso robo a Wells Fargo. Claridad (1992, junio 17) p. 19.

9 Mahony, E. Clinton Offer Includes Mastermind of 1983 Wells Fargo Robbery. Hartford Courant (1999, August 12) p. a.1. 
LAS AUTORIDADES ESTADOUNIDENSES Y LA LIBERTAD DE PRENSA

Incidental al proceso de los independentistas en Hartford, la prensa puertorriqueña sufrió también los desmanes de las autoridades federales. En agosto de 1991, la periodista Daisy Sánchez, enfrentaba a las autoridades estadounidenses al ser citada so pena de desacato por un Gran Jurado Federal en Hartford, para que entregara los originales de audio y video de las entrevistas hechas a los prófugos de la justicia Filiberto Ojeda y Luis Colón. Ante la negativa a entregar los documentos solicitados por el gobierno, la periodista enfrentaba amenazas de penas de hasta 12 años de cárcel.

Como respuesta, la directora del noticiario para el que trabajaba, los directores de otros noticieros locales, la Asociación de Periodistas de Puerto Rico, la Organización Internacional de Periodistas, el Club Ultramarino de Prensa y la Federación Latinoamericana de Trabajadores de Prensa, así como otros sectores como el Colegio de Abogados, el Movimiento Ecuménico Nacional de Puerto Rico, el Instituto Puertorriqueño de Derechos Civiles, organizaciones sindicales, senadores y los grupos de apoyo a los independentistas, entre otros, reiteraron su apoyo a la periodista. ${ }^{10}$

El abogado de Sánchez, Roberto Roldán logró una exitosa defensa del derecho de la prensa en el marco de la Primera Enmienda de la Constitución de los Estados Unidos, la cual garantiza la libertad de prensa y el principio ético de confidencialidad entre el periodista y su fuente. El juez federal estadounidense Emmet Clarie decidió relevar a Sánchez de su comparecencia ante el Gran Jurado. ${ }^{11}$

Una experiencia diferente al caso de Daisy Sánchez se experimentó en Puerto Rico cuando la periodista Maggie Bobb del rotativo EIMundo colaboró con las autoridades federales al trasmitirle como evidencia toda información que Los Macheteros le hicieron llegar. A pesar de que sus declaraciones ni implicaban o identificaban a alguno de los acusados, el tribunal encontró que dicho testimonio era valioso para efectos de establecer la veracidad de la secuencia de los hechos. La defensa de los acusados le preguntó si acostumbraba a contar al FBI sus conversaciones con sus fuentes. Bobb se limitó a contestar que ella estaba en libertad de decir lo que fuera si sus fuentes no le pedían confidencialidad. ${ }^{12}$

\section{LA PRENSA, LA PRENSA INDEPENDIENTE Y EL ROL DE LAS CAMPAÑAS DE APOYO}

En el plano político, tanto los operativos para los arrestos de los acusados por el robo de Connecticut, como el proceso judicial se pueden interpretar como un castigo con un doble propósito: reprimir el ala radical del independentismo. Así como procurar la "seguridad" y el "orden" ante el público en general o los observadores del operativo y su proceso judicial (Garland, 1999: 49-50, 71, 80).

En su ejercicio de poder, además de adjudicar responsabilidades, el Estado buscó construir o recuperar una imagen pública sobre su autoridad. Con la intención de consolidar su monopolio sobre la violencia como medio para respaldar dicha autoridad. También buscó suprimir la violencia subversiva por medio de un acto contundente, dirigido a desalentar el uso de la violencia por agentes sociales o políticos no estatales. Reafirmando su monopolio del poder coercitivo. Un paso subsiguien-

10 Cotto, C.. Firme defensa de la libertad de prensa. Claridad (1991, septiembre 13) p. 5.

11 Aquino, A. Decisión favorece libertad de prensa. Claridad(1991, diciembre 13). p.4.

12 Pastrana, M. Periodista daba información al FBI. Claridad (1989, marzo 10) p. 4; Pastrana, M. Por concluir juicio en Hartford. Claridad (1989, marzo 3) p.4. 
te, se dio al retirar dicha violencia de la arena pública mediante los procesos en el tribunal o la cárcel, en donde la violencia se impartió de forma impersonal. Medidas dirigidas a desalentar cualquier tipo de simpatía o empatía con los imputados.

A pesar de los logros por el Estado, los imputados lograron algunas victorias políticas cuando las barreras establecidas por el Estado, para disfrazar las prácticas violentas contra los imputados dentro de los tribunales y su reclusión en las cárceles, fueron combatidas por las campañas y publicaciones solidarias, apelando a combatir la indiferencia e inconciencia sobre la situación de los 15 independentistas. También al poder llamar la atención a la comunidad internacional sobre la situación política de Puerto Rico.

El procedimiento corriente de aislamiento de los presos del contacto público, y la limitación del conocimiento sobre las circunstancias de los arrestados, fue desafiado por la prensa independiente (específicamente el semanario Claridad) y los grupos de solidaridad con los presos políticos. Desafiando la influencia del Estado de los medios de información, o la reproducción del discurso "antiterrorista" del Estado. Por lo que la campaña de información por parte de estos grupos solidarios con los acusados, logró proveer medios alternos para incidir en la opinión pública mediada por el Estado.

Los logros en este renglón pueden ser difíciles de medir, cuando se utiliza como indicador o referencia lo que los principales medios de comunicación, como fuente de información histórica, reflejaron sobre la opinión pública. Sin embargo, los resultados del juicio de Filiberto Ojeda Ríos con su absolución por un jurado en Puerto Rico y el apoyo existente a las actividades y campañas de solidaridad demuestran la relevancia y las conquistas dentro de la dimensión política de los casos.

El despliegue de fuerza y los mensajes antiterrorista dirigida al público, tuvo diferentes efectos en Puerto Rico y en Connecticut. Manifiesto en los gestos de solidaridad con los imputados, y en las deliberaciones del jurado en los casos estudiados.

Tanto para el Estado como los acusados los logros o fracasos, dentro de la dimensión política del caso, fueron mixtos. El Estado tuvo que reconocer los motivos políticos de los delitos.

En cuanto a las imputaciones de terrorismo, las víctimas de Los Macheteros pertenecían a los cuerpos de seguridad del Estado. Los mismos cuerpos encargados de ejercer la violencia del Estado para procurar y preservar el orden público en la colonia. Los Macheteros lograron capturar la atención de los medios masivos de comunicación y hacer conocer al público los motivos políticos de sus acciones. Pero éstos enfrentaban el hecho de que defendían una ideología minoritaria dentro de una población políticamente dividida en la isla. Ante una población en los Estados Unidos continentales altamente prejuiciada e ideológicamente muy conservadora. Por lo que los alcances en la educación o simpatía política, se limitaba casi por exclusivo a los adeptos de antemano a la ideología y métodos de los perpetradores.

En el lento transcurso del proceso criminal de las autoridades contra los 15 independentistas, los imputados tuvieron finalmente que adoptar el fraccionamiento organizativo para enfrentar los diferentes cargos radicados, obligándole a establecer diferentes estrategias de defensa legal, así como participar de diferentes campañas de apoyo y de divulgación mediática.

El fraccionamiento experimentado dentro del grupo de imputados, se puede entender por un lado, como una de las primeras victorias del Estado. Al traer a los independentistas imputados a su "terreno de juego" el Estado obligaba a los imputados a asumir una actitud defensiva, teniendo que ampararse en las reglas de juego es- 
tablecidas por las autoridades. El estado de derecho y los tribunales. Lo que tradujo para los independentistas acusados en una perdida considerable de terreno político.

Por otro lado, el fraccionamiento puede interpretarse como una estrategia o mera necesidad práctica de los imputados, para contrarrestas las ventajas del Estado en su arena.

Los medios físicos en los que desarrollaron Los Macheteros y el Estado sus acciones, fueron muy distantes. Sin embargo, es en los espacios donde fueron procesados legalmente y el espacio mediático desde donde se informó de los procesos legales, fueron los espacios públicos en que ambos actores llevaron a cabo su último combate: debatir la legalidad como la legitimidad de sus actividades ante la opinión pública.

Tomando el balance del duelo habido entre el Estado y los acusados en el caso de la Wells Fargo, en su mayoría los acusados alcanzaron ciertas victorias al obtener absoluciones; poder negociar las sentencias, lograr clemencias presidenciales, lograr el reconocimiento de los motivos políticos de sus delitos y no tener que dar cuenta por los $\$ 7$ millones. Ni dar cuenta sobre los demás operativos de Los Macheteros. Sin embargo, lo anterior encontró una dramática excepción con el asesinato de Filiberto Ojeda por el FBI, el 23 de septiembre de 2005 (Comisión de Derechos Civiles, 2011). En una nueva coyuntura de la guerra de los Estados Unidos contra el terrorismo, el Estado seguía reafirmando su capacidad coercitiva como medio de control social.

\section{Comentarios finales}

El rol de la prensa escrita en la cobertura de los acontecimientos de los operativos clandestinos de Los Macheteros, sus arrestos y procesamiento judicial, fue fundamental. No solamente en la forma en que se levantó una relación de los acontecimientos. Esencial en el ejercicio historiográfico de investigar lo acontecido en el caso de Los Macheteros. También en la forma en que el periodista se convirtió en protagonista de los procesos, cuando las autoridades intervinieron con su actividad periodística y estos tomaron posturas. Ceder ante los aparatos coercitivos del estado. 0 defender el derecho a la libertad de prensa.

\section{Referencias Bibliográficas}

Comisión de Derechos Civiles. (2011). Informe final sobre la investigación de los sucesos ocurridos en el Municipio de Hormigueros el 23 de septiembre de 2005 donde resultó muerto el ciudadano Filiberto Ojeda Ríos.

Fernández, R. (1993). Los Macheteros: el robo a la Wells Fargo y la lucha armada por la independencia de Puerto Rico. Río Piedras, PR: Editorial Edil.

Garland, D. (1999). Castigo y sociedad moderna. Un estudio de teońa social. Argentina: Editores Siglo Veintiuno.

U.S. Attorney's Office (2010, May 26) Avelino Gonzalez-Claudio Sentenced to Seven Years in Federal Prison for Role in 1983 Wells Fargo Robbery. Retirado de https://www.fbi.gov/ newhaven/press-releases/2010/nh052610c.htm

U.S. Attorney's Office (2012, November 14) Norberto Gonzalez-Claudio Sentenced for Role in 1983 Wells Fargo Robbery, Illegal Possession of Machine Gun. Retirado de https:// www.fbi.gov/newhaven/press-releases/2012/norberto-gonzalez-claudio-sentencedfor-role-in-1983-wells-fargo-robbery-illegal-possession-of-machine-gun 